\title{
1An improved primer set and amplification protocol with increased specificity and 2sensitivity targeting the Symbiodinium ITS2 region
}

3Benjamin CC Hume ${ }^{1} \dagger$, Maren Ziegler ${ }^{1}$, Julie Poulain ${ }^{2,3,4}$, Xavier Pochon ${ }_{-}^{35,6}$, Sarah Romac ${ }_{-}^{47}$, 4Emilie Boissin $n_{-}^{58}$, Colomban de Vargas. 5 Voolstra $^{1} \dagger$

$6^{1}$ Red Sea Research Center, Bivision of Biølogieal Seienees and Engineering, KAUSTKing 7 Abdullah University of Science and Technology, Thuwal__23955-6900, Saudi Arabia

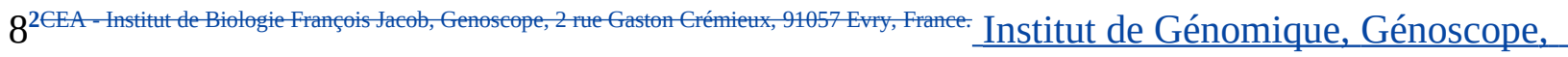
9Evry, France

$10^{3}$ ENRS, UMR 8030, EP5706, Evy, Franee.

114'Université d'Evy, UMR 8030, CP5706, Evy, France.

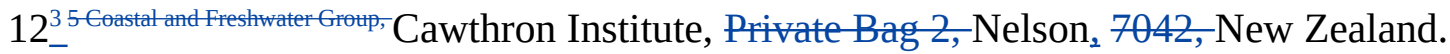

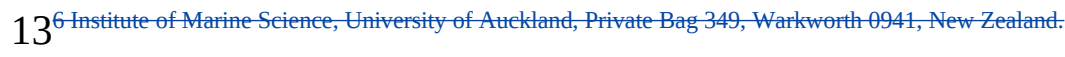

14-- CNRS, UMR 7144, EPEP \& Sorbonne Universités, UPMC Université Paris 06; Station 15Biologique de Roscoff, z9680-Roscoff, France.

$16_{-}^{58}$ PSL Research University: EPHE-UPVD-CNRS, USR 3278 CRIOBE, Université de Perpignan, 1752 Avente Paul Alduy, 66860-Perpignan Cedex, France

18

19†Corresponding authors:

20Christian R Voolstra

21Bldg 2, Rm 2226 
22Red Sea Research Center, KAUST

23Phone: +966-12-808 2377

24Email: christian.voolstra@kaust.edu.sa

25

26Benjamin C C Hume

27Bldg 2, Rm 2227 WS01

28Red Sea Research Center, KAUST

29Phone: +966-544-701-291

30Email: benjamin.hume@kaust.edu.sa

31

32Running title: Assessment of Symbiodinium ITS2 primers pairssets-

33Keywords: Coral Reef, Metabareoding, Metagenomies, Next-generation sequeneing, Mierobial34Biology, Symbiodinium 


\section{Abstract}

36The Internal Transcribed Spacer 2 (ITS2) rRNA gene is a commonly targeted genetic marker to 37assess diversity of Symbiodinium, a dinoflagellate genus of algal endosymbionts that is 38pervasively associated with marine invertebrates, and notably reef-building corals. Here we tested 39three commonly used ITS2 primer pairs (SYM_VAR_5.8S2/SYM_VAR_REV, 40ITSintfor2/ITSReverse, and ITS-DINO/ITS2Rev2) with regard to amplification specificity and 41sensitivity towards Symbiodinium, as well as sub-genera taxonomic bias. We tested these primers 42over a range of sample types including three coral species, coral surrounding water, reef surface 43water, and open ocean water to assess their suitability for use in large-scale next generation 44sequencing projects and to develop a standardized PCR protocol. We found the 45SYM_VAR_5.8S2/SYM_VAR_REV primers to perform superior to the other tested ITS2 46primers. We therefore used this primer pair to develop a standardised PCR protocol. To do this, 47we tested the effect of PCR-to-PCR variation, annealing temperature, cycle number, and different 48polymerase systems on the PCR efficacy. The Symbiodinium ITS2 PCR protocol developed here 49delivers improved specificity and sensitivity towards Symbiodinium with apparent minimal sub50genera taxonomic bias across all sample types. In particular, the protocol's ability to amplify 51Symbiodinium from a range of environmental sources will facilitate the study of Symbiodinium 52populations across biomes. 


\section{Introduction}

54Coral reefs sustain some of the highest levels of biodiversity on Earth and provide a range of 55services to communities totalling millions of people (Moberg \& Folke 1999; Plaisance et al. 562011). However, these ecosystems are being lost at an alarming rate (Hughes et al. 2017; 57Norstrom et al. 2016). This loss is primarily due to anthropogenic stressors degrading the 58scleractinianSeleraetinian corals that build and support these reefs. Interestingly, susceptibility to 59these stressors amongst reef-building corals is often not homogenous (Kemp et al. 2014; Pandolfi 60et al. 2011; Rowan et al. 1997).

61Coral resilience is determined not only by the animal genotype but also by the diversity of 62microbes that associate with the animal host (Bourne et al. 2016; Levin et al. 2017; Peixoto et al. 632017; Pogoreutz et al. 2017; Radecker et al. 2015; Torda et al. 2017; Ziegler et al. 2017c). Of the 64microbial components that make up the coral holobiont - the consideration of the animal host and 65its associating microbes as a functional ecological unit - the algal symbionts of the genus 66Symbiodinium have received the most attention for their role in affording resilience to the coral 67host (Hume et al. 2016; Thornhill et al. 2017). The coral-Symbiodinium association is generally 68obligate with the algal symbiont providing towards the nutritional needs of the animal host, in 69exchange for a stable, beneficial environment (Muscatine 1990). The efficacy and character of 70this association is- determined by the genotype of alga (Kemp et al. 2014; Rowan et al. 1997; 71Silverstein et al. 2015; Thornhill et al. 2017). Accordingly, the ability to resolve taxa within the 72genus Symbiodinium is essential to better understanding resilience of the coral holobiont and reef 73ecosystems as a whole. 
74Taxonomic resolution within Symbiodinium is primarily achieved genetically (Wham et al. 2017). 75To this end, a range of genetic markers and associated analytical approaches exist (Pochon et al. 762014; Thornhill et al. 2017). Initially, genetic characterisations resolved Symbiodinium into broad 77taxonomic groupings referred to as clades A-I (Pochon \& Gates 2010). However, due to the 78significant genetic and phenotypic diversity found within these clades, contemporary genetic 79resolutions are conducted at a sub-cladal level. For such analyses, the most commonly used 80marker is the ITS2 region of the nrDNA (LaJeunesse 2001). This marker is multi-copy in nature 81meaning that a single Symbiodinium cell may contain 100s-1000s of copies of the ITS2 region 82(Arif et al. 2014; LaJeunesse 2002). As such, 100s of distinct ITS2 sequences may be generated 83from a single genotype, referred to as intragenomic diversity. Given that corals may associate 84with multiple Symbiodinium genotypes, intergenomic diversity may also exist in samples. 85Disentangling these two diversities can complicate analyses, but ultimately, the intragenomic 86diversity of the ITS2 region has proven to be a rich source of information that has been 87effectively leveraged to improve resolutions within Symbiodinium (Smith et al. 2017; Wham et 88al. 2017).

89Symbiodinium ITS2 intragenomic diversity was first employed taxonomically using denaturing 90gradient gel electrophoresis (DGGE) methodologies, enabling the separation of PCR amplicon 91sequence fragments according to melting temperature, a proxy for sequence (LaJeunesse 2002). 92Using such techniques, Symbiodinium taxa that shared the same most abundant ITS2 sequence in 93common, yet displayed significantly different functional phenotypes, were able to be resolved 94specifically according to differences in rarer sequences in their intragenomic diversity. 95Importantly, Symbiodinium taxa began to be resolved in part bypartby specific sets of these 
96intragenomic ITS2 sequences referred to as ITS2 types or profiles. For example, S. trenchii and 97S. glynnii have the D1 ITS2 sequence in common but can be differentiated according to different 98presence-absence combinations of the D4, and D6 sequences (Wham et al. 2017). Similarly, 99S. thermophilum, a symbiont prevalent in corals living in the world's warmest reefs, can be 100differentiated from other C3 dominated symbionts, that have a global distribution, through the 101identification of a specific intragenomic sequence, S. thermo.-indel (Hume et al. 2015).

102More recently, studies have been taking advantage of the sequencing benefits afforded by next 103generation sequencing (NGS) of PCR amplicons to elucidate Symbiodinium diversity using the 104ITS2 region. Multiple approaches to dealing with the sequence diversity have been taken, 105including, but not limited to, OTU clustering (Arif et al. 2014; Cunning et al. 2017; Ziegler et al. 1062017a; Ziegler et al. 2017b), searching for specific taxa-defining ITS2 sequences (Boulotte et al. 1072016; Hume et al. 2016; Ziegler et al. 2017a) or looking for ITS2 profiles found in common 108between samples (Smith et al. 2017).

109Given the ongoing surge in Symbiodinium diversity studies employing NGS approaches using the 110ITS2 marker, and the large-scale projects that rely on them, such as The Global Coral 111Microbiome Project (http://vegathurberlab.oregonstate.edu/global-coral-microbiome-project) and 112the TaraTARA Pacific expedition (http://oceans.taraexpeditions.org/en/m/environment/ocean113climate/new-expedition-tara-pacific/), we sought to establish, and future proof, a standardised and 114improved PCR protocol. Particularly, we assessed the specificity (preferential amplification of 115Symbiodinium DNA), sensitivity (ability to amplify Symbiodinium from Symbiodinium-rare 116environments), and relative sub-genera taxonomic bias (within Symbiodinium bias) of existing 117ITS2 primer pairs, as well as the effect of different polymerase systems. Notably, we tested 
118amplification on a range of sample types including coral, coral surrounding water, reef surface 119water, and open ocean water due to increasing efforts to elucidate environmental reservoirs and 120assess free-living Symbiodinium (Mordret et al. 2016; Nitschke et al. 2016; Pochon et al. 2010; 121Thornhill et al. 2017). 


\section{Materials \& Methods}

\section{Sample collection, DNA isolation, ITS2 primer pairs, and experimental setup}

124To establish a robust ITS2 NGS-based amplification protocol for Symbiodinium, we assessed the 125effectiveness of three commonly used primer pairs (Table 1) on samples from different 126environments (Table 2). Further, we tested the robustness of the most effective primer pair, 127SYM_VAR, by documenting the effects of PCR-to-PCR variation, annealing temperature, cycle 128number, and polymerase system used with regards to: specificity, sensitivity, relative sub-genera 129amplification, and which sequences were returned. Chimeric sequence formation was 130additionally assessed as a function of PCR cycles.

131Sample collection: In total, 27 samples were used in our analyses from 4 different environments: 132coral tissue (CO), coral surrounding water (CSW), coral reef surface water (SRF-CO), and open

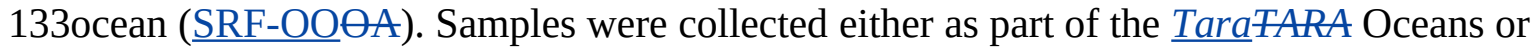
134TaraTARA Pacific expeditions. Field Permits were obtained as part of the TaraTARA Oceans and 135TarafARA Pacific expeditions and the approving bodies were the respective governments and/or 136ministries for the environment of the countries where the samples were collected. The authors of 137this work are part of the TarafARA Oceans and TarafARA Pacific expeditions, and thus, 138participated in sample collection. Tara Expeditions are global scientific voyages aimed at 139probing morphological and molecular diversity, evolution and ecology of marine plankton 140(viruses, bacteria, archaea, protists and planktonic metazoans) in the photic layer of the world 141oceans to research how they are impacted by Earth's climate changes. Tara meteorological, 142oceanographic, biochemical, and plankton morphological data are archived in the comprehensive 
143Sample Registry of the PANGAEA database (https://www.pangaea.de).

144(https:/tpangaea.de). The Sample Registry links physical samples to metadata about

145sampling and analysis methodology performed on each sample. Whilst the Tara Pacific registry

146is still under construction at the time of writing of this manuscript, the Tara Oceans registry can

147be accessed at https://doi.pangaea.de/10.1594/PANGAEA.842237.

148Six CO samples from three genera, 2 Porites lobata (samples: CO-0000150 and CO-0000151), 2

149Pocillopora meandrina (samples: CO-0000208 and CO-0000209),; and 2 Millepora platyphylla

150(samples: CO-0000302 and CO-0000303),; were collected on SCUBA off the coast of Panama in

151July 2016. Corals were stored in DNA/RNA Shield (Zymo Research, USA).

152Two CSW and one SRF-CO samples were also collected off the coast of Panama in July 2016.

153CSW samples were collected (15-17 L) from water surrounding 2 Pocillopora corals using a

154boat-mounted vacuum pump. SRF-CO samples were collected $(18 \mathrm{~L})$ directly from the surface of

155the reef. Water was pre-filtered through a $20 \mu \mathrm{m}$ metallic sieve, and then vacuum filtered on a 3

156 $\mu \mathrm{m}$ polycarbonate filter of $142 \mathrm{~mm}$ diameter for $15 \mathrm{mins}$. After filtration, filters were

157immediately flash frozen in liquid nitrogen.

158In addition, six SRF-OOOA samples were collected off the coast of Panama and were processed

159as described above for the CSW and SRF-CO samples. Twelve additional samples were collected

160as part of the Tara Oceans expeditions TARA Oeean from a range of ocean basins including the

161Pacific, North Atlantic, and Indian Ocean. These samples were filtered to a size fraction of 5-20

$162 \mu \mathrm{m}$ as described in section 6a of Pesant et al. 2015.

163DNA isolation: DNA was extracted using the ZR-Duet DNA/RNA MiniPrep Plus (Zymo

164Research, USA) kit for all coral samples. Further, DNA was extracted from all water samples as 
165described in AlbertiAlerti et al.- (2017)(Alberti et al. 2017) for the 5-20 $\mu \mathrm{m}$ and 3-20 $\mu \mathrm{m}$ 166fractions. In brief, the protocol was based on simultaneous extraction of DNA and RNA by 167cryogenic grinding of cryopreserved membrane filters, followed by nucleic acid extraction with 168NucleoSpin RNA kits (Macherey-Nagel, Germany) combined with DNA Elution buffer kit 169(Macherey-Nagel, Germany).

170ITS2 primer pairs: Three pairssets of published ITS2 primer pairs specifically designed to 171amplify Symbiodinium DNA were tested. These were:

172SYM_VAR_5.8S2/SYM_VAR_REV(Hume et al. 2013; Hume et al. 2015), ITSintfor2/ITS173reverse (Coleman et al. 1994; LaJeunesse 2002), and ITS-DINO/ITS2Rev2 (Pochon et al. 2001; 174Stat et al. 2009). Primer details and amplification protocols are given in Table 1. For ease of 175comprehension, each primer pair will be referred to as the SYM_VAR, ITSintfor2, or ITS-DINO 176primer pair.

177Experimental setup: Sequencing for this study was conducted in two consecutive efforts. In the 178first part, aimed at directly comparing the three primer pairs, each primer pair was used to 179amplify each of the 27 samples. In the second part, a subset of samples was amplified using the 180SYM_VAR primer pair with different PCR protocols. We tested the effect of number of cycles, 181annealing temperature, and polymerase system on selectivity, sensitivity, and stability to optimise 182the reaction. Details of the samples as well as the associated number of cycles and annealing 183temperatures used can be found in Table 2 and Supplementary Table 1. All PCRs were carried 184out in $25 \mu \mathrm{l}$ reactions with the following conditions: $12.5 \mu \mathrm{l}$ Phusion High-Fidelity PCR Master 185Mix 2X (ThermoFisher Scientific, USA) or equivalent amount of Advantage 2 PCR system 186(Takara Bio, Japan), $1 \mu \mathrm{l}$ of forward and reverse primer at a concentration of $10 \mu \mathrm{M}, 0.75 \mu \mathrm{l}$ of 
187DMSO, $1 \mu \mathrm{l}$ of genomic DNA at a concentration of between 5-10 ng and $8.75 \mu \mathrm{l}$ of $\mathrm{ddH}_{2} 0$ with a 188GeneAmp PCR system 9700 (Perkin Elmer, USA).-

\section{Library preparation, sequencing, and sequence quality control}

190For both sequencing efforts, all PCRs were conducted in triplicate before being pooled for 191sequencing. Pooled PCR products were purified using 0.8 x AMPure XP beads (Beckmann 192Coulter Genomics, USA), then aliquots of purified amplicons were run on an Agilent Bioanalyzer 193(Agilent technologies, USA) using the DNA High Sensitivity LabChip kit to verify lengths and 194were quantified using a Qubit Fluorometer (ThermoFisher Scientific, USA).

195One hundred ng aliquots of the amplicons were directly end-repaired, A-tailed, and ligated to 196Illumina adapters (Illumina, USA) on a Biomek FX Laboratory Automation Workstation 197(Beckman Coulter, USA). Then, library amplification was performed using the KAPA Hifi 198HotStart NGS kit (KAPA Biosystems, USA). After library profile analysis by LabChip GX 199(Perkin Elmer, USA) and qPCR quantification using PicoGreen (ThermoFisher Scientific, USA) 200ønin 96-well plates, each library was sequenced using 250 bp read length chemistry in a paired201end flow cell on a MiSeq instrument (Illumina, USA).

202During sequencing, Illumina's Real Time Analysis (RTA) was run with default settings to 203remove clusters with the least reliable data. Output BCL files were converted to demultiplexed 204fastq files using Illumina's bcl2fastq package and in-house filtering was applied to reads that 205passed the Illumina quality filters (Alberti et al. 2017). Read pairs that mapped onto run quality 206control sequences (Enterobacteria phage PhiX174 genome) were removed using the bowtie 207package. 
208For each of the sequenced samples analysed in this study the same sequence quality control (QC) 209was performed. Briefly, mothurmother_1.39.5 (Schloss et al. 2009) was used to create contigs 210from paired forward and reverse demultiplexed fastq.gz files using the make.contigs command. 211The screen.seqs command was then used (maxambig=0, maxhomop $=5$ ) to discard sequences 212putatively generated from sequencing errors. The unique.seqs command was used to create a non213redundant collection of sequences represented by a .name and ffasta file. The remainder of the 214QC was performed using both the .name and .fasta files produced. The pcr.seqs command 215 (pdiffs $=2$, rdiffs $=2$ ) was used to trim the primer sequence regions from the returned sequences. In 216addition, this command was used to discard sequences in which the specific primer pairs could 217not be found - indicative of poor sequencing quality - allowing a deviation of no more than 2 218nucleotides differences in either of the forward or reverse primer sequences. Next, split.abund 219was run (cutoff $=2$ ) to discard sequences that were not found more than 2 times in each sequenced 220sample - again, to reduce incorporating sequences with sequencing errors. Sequences were once 221again made non-redundant using the unique.seqs command. Finally, the summary.seqs command 222was used to calculate the size distribution of the remaining sequences. Sequences below the $2.5 \%$ 223and above the $97.5 \%$ size percentiles were removed using the screen.seqs minlength and 224maxlength parameters. A summary of the number of sequences retained at three stages of the QC 225process (pre-QC, post-QC, and Symbiodinium only sequences) are given in Supplementary Table 2261 and Supplementary Fig. 1.

\section{Sequence data analysis - primer pair comparisons}

228To assess which of the three primer pairs performed the best, each primer pair was assessed for 229specificity (i.e., preferential amplification of Symbiodinium DNA) and sensitivity (i.e., ability to 
230amplify Symbiodinium from Symbiodinium-rare environments). Additionally, potential within231Symbiodinium taxonomic biases were assessed by comparing the proportions of sequences for 232each primer pair that belonged to each of the 9 major cladal groups of Symbiodinium, clades A-I 233(Pochon \& Gates 2010). Our assumption was that if no or only small eladalclade biases exist, the 234proportion of sequences from each clade should be similar between primers. Consequently, a 235large deviation in eladatclade proportions by any one of the primer pairs- was regarded as 236indicative of taxonomic bias.

237The specific PCR conditions used for each primer pairset are given in Table 1 with PCR reagents 238as detailed above. The sequences returned from each PCR were annotated using blastn and the 239NCBI 'nt' database according to their closest match to one of the following categories:

240Symbiodinium, dinoflagellate, stony coral, Hydrozoan, uncultured eukaryote, other, or 'no 241match'. To do this, the ffasta file produced from the initial QC from each sample was run against 242NCBI's 'nt' database with the max_target_seqs argument set to 1 and an output format string of 243'6 qseqid sseqid staxids sscinames sblastnames evalue'. For sequences to be categorised as 244Symbiodinium, an e-value $>50$ was required (representing approximately $a-100 \%$ coverage 245 match at $80-85 \%$ nt identity) in addition to the closest match being of Symbiodinium origin. 246Additionally, closest match subject sequences were screened for two sequences in particular 247(JN406302 and JN406301), which are mis-annotated as Symbiodinium (highly divergent from 248any other Symbiodinium sequences) in the 'nt' database. Thus, query sequences matching these 249sequences were annotated as 'Unclutured eukaryote'. Notably, before controlling for this, $58 \%$ of 250all 'Symbiodinium' sequences amplified by the ITSintfor2 and ITS-DINO primer pairs ( $0 \%$ for 
251the SymVar primer pair) in the SRF-OOOA samples matched these sequences as their closest 252match (Supplementary Fig. 21).

253blastn was also used to associate Symbiodinium sequences to one of the 9 eladal groupsclades. To 254do this, a .fasta file was created for each sample that contained all sequences that had previously 255been categorised as Symbiodinium. This file was used as an input for blastn with the 256max_target_seqs argument set to 1 and an output format string of '6 qseqid sseqid evalue pident 257gcovs'. A custom BLAST database was used that contained a single eladal-representative 258sequence for each of the 9 clades (Supplementary Data 1).

\section{Sequence data analysis - optimizing the SYM_VAR PCR protocol}

260Having determined SYM_VAR as the most effective primer pair for the amplification of the 261Symbiodinium ITS2 region, we undertook a second sequencing effort to optimize the PCR 262protocol. Specifically, we investigated: 1) how consistent the PCR was (i.e. PCR-to-PCR effect) 263by conducting PCRs with the exact same reaction conditions but sequenced enin different 264sequencing runs, 2)), whether increasing the annealing temperature could increase sensitivity in

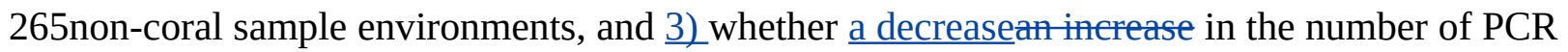
266cycles would still allow for a satisfactory actually aids in the-amplification of Symbiodinium 267whilst decreasingfrom samples representing Symbiodinium-rare enviromments. Cladal variation

268and quantity of chimeric sequences return. formation were additionally considered, when 269investigating the effects of increased annealing temperatme and number of PCR eycles.

270PCR conditions were the same as for the SYM_VAR_5.8S2/SYM_VAR_REV primer pair 271(Table 1), but with a 30 sec denaturing step, following the manufacturer's recommendation_ 272(Phusion High Fidelity PCR; ThermoFisher Scientific, USA).- Taxonomic identities and the- 
273 ladalclade breakdown (Symbiodinium sequences only) of sequences returned from each sample 274(annealing temperature and cycle number comparisons only) were determined in the same way as 275for the initial primer pairset comparisons described above.

276As a final optimisation tøof the SYM_VAR amplification protocol, we tested whether the 277polymerase enzyme system used could affect the outcome of the PCR amplification. For 11 278samples (Table 2), we contrasted amplification with Phusion High-Fidelity PCR Master Mix 279(ThermoFisher Scientific, USA) and the Advantage 2 PCR Enzyme System (Takara Bio, Japan). 280PCR amplification with the Advantage 2 PCR Enzyme System were conducted as shown in 281Table 1, but with 30 cycles.

282To further assess the potential effect of PCR protocol modifications, we additionally assessed 283whether PCR-to-PCR variation, or any of the protocol optimisation modifications would affect 284which sequences were amplified. To assess this, the top 20 most abundant sequences (by relative 285abundance) were identified and plotted by decreasing abundance for pairings of sequenced 286samples for which PCR conditions were identical, or only differed in one of the investigated 287parameters (i.e., cycle number,ғ annealing temperature, or polymerase system). This analysis was 288conducted for two samples, AW-0000035 and IW-0000015 and only for Symbiodinium 289sequences contained in each. It should finally be noted that although we did not test for it here, 290variability between different PCR cyclers (e.g. in ramping rate and accuracy of temperature 291binning) also has the potential to cause variation in a given PCR. 292An online version of the final optimized PCR protocol is available through the doi: 293 dx.doi.org/10.17504/protocols.io.n8edhte.

2945. Design of the SYM VAR primer pair 
295The SYM VAR primer pair were designed to minimise eladatclade bias and coral-host 296complementarity. Originally, the reverse primer of the SYM VAR primer pair that binds in the 29728 S region of the rDNA, SYM VAR REV, was designed to be complimentary to the forward 298primer SYM VAR FWD that binds in the 18S (Hume et al. 2013). This primer pair was 299designed to amplify both ITS regions as well as the 5.8S region of the rDNA. These primers were 300designed with the incorporation of degenerate bases based upon a multiple sequence alignment 301containing representatives of all Symbiodinium clades so that, where possible, the primer 302sequences had an equal number of nucleotide mis-matches to each of the clades. Later, to be able 303to amplify only the ITS2 region of the rDNA, the SYM VAR 5.8SII primer was designed 304(Hume et al. 2015). Compatibility to the SYM VAR REV primer and a minimisation of 305 eladalclade bias were maintained whilst complementarity to coral host ITS2 rDNA sequences 306 was also taken into account by including representative sequences from $P$. lobata and $P$. lutea 307(ITS2 sequences from which, had been amplified using alternative primers) to the multiple 308sequence alignment (Hume 2013). It should be noted that this primer pair was successfully used 309to recover Symbiodinium DNA from Acropora downingi, Cyphastrea microphthalma, Favia 310pallida, Platygyra daedalea, Porites harrisoni, and P. lutea in Hume et al (2015).

\section{Sampling permits}

312The following permits are were required for the collection of the samples analysed as part of this 313study: Panama scientific sampling: SE/AP-18-16; Panama CITES: SEX/A-72-16, no. 05567; 314PortgatPortugal scientific sampling: 080522/2009/Proc. ${ }^{\circ}$ E.17.b; Maldives scientific sampling: 315FA-D2/33/2010/02; Chile scientific sampling: 13270/24/74. 


\section{Results}

\section{Primer pair comparisons for amplification of Symbiodinium ITS2}

318In the CO samples, the SYM_VAR primer pair -exclusively amplified Symbiodinium DNA. For

319the ITSintfor2 and ITS-DINO primer pairs Symbiodinium sequences represented 84\% (SD 11\%)

320and 79\% (SD 12\%) of total reads, respectively. Remaining sequences associated with

321Scleractinia, Hydrozoa, or resulted in no BLAST match (Fig 1).-

322In the CSW water samples, the SYM_VAR primer pair maintained a high specificity for 323Symbiodinium (98\%; SD 3\%). In contrast, the alternative pairs amplified 51\% (SD 23\%) and 32442\%_SD 24\%) of Symbiodinium reads, respectively, with the remaining sequences characterised 325as dinoflagellate or falling into the uncultured eukaryote category (Fig 1).

326In the SRF-CO sample, Symbiodinium amplifications were considerably lower compared to the 327CO and CSW samples. SYM_VAR amplified 43\% of Symbiodinium reads compared to $1 \%$ in 328both alternative primer pairs. The remaining sequences in the SYM_VAR pair were primarily of 329dinoflagellate origin. In the other two primer pairs, although a large proportion were also 330dinoflagellate in origin, sequences primarily fell into the uncultured eukaryote category (Fig 1).

331In the SRF-OOOA samples, the ITSintfor2 and ITS-DINO primer pairs were more similar in 332their amplifications compared to the SYM_VAR pair. In contrast to the other primer pairs, 333SYM_VAR amplified less 'uncultured eukaryotic', 'other', and 'no match' sequences, and more 334dinoflagellate and Symbiodinium sequences. Specifically, amplification of Symbiodinium was 335achieved in 12, 7 and 8 of the SRF-OOӨA samples using the SYM_VAR, ITSintfor2 and ITS336DINO primer pairs, respectively. All of the 5 libraries that achieved a Symbiodinium 
337amplification above 5\% of total reads were amplified with the SYM_VAR primer pair. In total, 338the SYM_VAR primer pair achieved a Symbiodinium amplification of 7\% (SD 15\%)compared 339 to $<0.5 \%$ (SDs $<0.5 \%$ ) for the alternative primer pairs (Fig 1).

340Across all samples in which all three primer pairs amplified $>5 \%$ of Symbiodinium reads, the 341proportions of sub-genus taxa amplified (Symbiodinium clades) were comparable (Fig 1).

\section{Establishment of an optimal PCR protocol using the SYM_VAR primer pair}

343First, we assessed whether the same Symbiodinium ITS2 sequences were returned from PCRs 344performed on the same samples, with identical reaction conditions, but sequenced enin different 345sequencing runs. For the two samples tested, the 20 most abundant sequences were present in 346both PCRs pairings with relative abundances of each sequence being comparable (Figure 2).

$347 \mathrm{Next}$, we increased the annealing temperature from $56^{\circ} \mathrm{C}$ to $59^{\circ} \mathrm{C}$ to see whether a gain in 348Symbiodinium selectivity, and therefore sensitivity, could be achieved without yielding sub-genus 349taxonomic bias. In the CSW and SRF-CO samples, increasing annealing temperature increased 350the proportion of returned Symbiodinium sequences from 95 to $98 \%$ and from 28 to 47\%, 351respectively. However, in both samples a small increase in the proportion of clade D versus clade 352C was observed, 45 to $47 \%$ and from 57 to $66 \%$, respectively. In the two SRF-OOӨA samples 353tested, no Symbiodinium amplification occurred at either $56^{\circ} \mathrm{C}$ or $59^{\circ} \mathrm{C}$ (Fig 3). The 20 most 354abundant sequences were found in both libraries for the CSW samples tested. However, 2 of the 35520 sequences were missing from one of the libraries in the SRF-CO sample tested (the $10^{\text {th }}$ and $35616^{\text {th }}$ most abundant sequences; Figure 2). 
357Additionally, we tested whether Symbiodinium could be sufficiently amplified using a decreased 358an increase in the-number of PCR cycles aetually improves the amplifieation of Symbiodinium 359from samples representing Symbiodinium-rare environments without leading to reducean inerease 360in chimeric sequence formation or taxonomic bias. To accomplish this, we compared by 361 eomparing the above-used protocol to one with onlya reduced number of 25 cycles. In the CSW 362and SRF-CO samples there was a greateran increase in the proportion of Symbiodinium amplified 363at 35 compared to 25 cycles ( 5\% greaterinerease in the CSW samples and 15\% in the SRF-CO 364samples). However, in both of the SRF-OOӨA samples no Symbiodinium amplification was seen 365atin either of the-cycle conditions. Only non-Symbiodinium, dinoflagellate taxa sequences were 366amplified. Of the 9 samples considered in the chimeric analysis (the $\underline{3}$ three CSW and SRF-CO 367samples considered above, as well as $\underline{6}$ six CO samples) greater number ofan increase in predicted 368chimeric sequences were putatively identified was observed at 35 cycles versus 25 cycles in 3 of 369them with an increase of $1 \%$ in each case (Table 3). The proportions of sub-genus taxa amplified 370(Symbiodinium clades) were comparable (Fig 3) and all but one (the $20^{\text {th }}$ ) of the most abundant 371sequences were amplified in common (Fig 2).

372Last, we also tested to see whether there would be any effect of using different polymerase 373systems. In the CO and SRF-OOOA samples tested, no difference in proportion of returned 374Symbiodinium sequences was observed between polymerase systems used $(100 \%$ and $0 \%$ 375amplification for all libraries, respectively). However, in the CSW and SRF-CO samples we 376observed a relative decrease of Symbiodinium reads in the libraries that used the Advantage 2 377system (Fig. 4). Despite, this relative decrease, the polymerase system used had minimal effect 378on which Symbiodinium sequences were returned (top 20 most abundant; Fig 2). 


\section{Discussion}

380NGS approaches are quickly superseding their traditional gel-based counterparts due to advances 381in sequencing power and ease of handling (Arif et al. 2014; Thomas et al. 2014). In particular, 382increases in sequencing depths by several orders of magnitude havehas enabled the examination 383of low-abundance ITS2 sequences for the first time. The illumination of these rare-sequences 384holds the potential to characterising the rare Symbiodinium biosphere in hospite and in free-living 385contexts (e.g. Boulotte et al. 2016; Mordret et al. 2016; Ziegler et al. 2017b). Also, the 386incorporation of rarer ITS2 sequences into phylogenetic analyses has the potential to vastly 387improve the taxonomic resolving power of the ITS2 marker (Smith et al. 2017).

388As rarer ITS2 sequence variants become more integral to the biological inferences made 389(Boulotte et al. 2016; Ziegler et al. 2017b), the effect of differences in ITS2 amplification 390strategies will become more significant. For example, small changes in amplification strategy 391may have a large effect on the rarer sequence variants returned. Despite this potential sensitivity 392to amplification variation, NGS studies published to date have used a range of different primer 393pairs developed for use in less-sensitive analytical contexts, without first assessing the effect of 394primer choice. For example, ineffective amplifications have the potential to compromise our 395ability to infer taxonomic identities, and inter-primer pair variation could hinder cross-study 396comparisons. In particular, since several large-scale projects including the Global Coral 397Microbiome Project and TaraTARA Pacific, in which thousands of samples are being analysed 398for Symbiodinium ITS2 diversity, many of which represent Symbiodinium-rare environments, it is 399important to standardise and test proof the primer pair and PCR protocol employed. Here, we 
400compared three commonly used Symbiodinium ITS2 primer pairs, and show that the SYM_VAR 401primer pair outperforms the two alternative pairs, in specificity and sensitivity whilst maintaining 402an apparent minimal taxonomic bias.

403Our data show that primer pair had a considerable effect on amplification efficacy of the

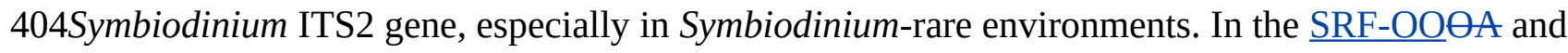
405SRF_CO samples, the SYM_VAR primer pair significantly outperformed the alternative primers 406amplifying more Symbiodinium reads in every sample tested (Fig 1). Only SYM_VAR were able 407to amplify Symbiodinium in the majority of SRF-OOӨA samples with proportions as high as $40846 \%$. In contrast, the maximum amplification of the alternative primers was $2 \%$ with both pairs 409returning averages of $<1 \%$ from successful amplifications in less than half the samples. In more 410Symbiodinium-abundant samples, such as the CO or CSW samples, the use of the SYM_VAR 411primers enabled a significant gain in the proportion of Symbiodinium sequences returned, once 412again amplifying more Symbiodinium in every sample tested (Fig 1). In practical terms, this 413translates to needing a shallower sequencing effort per sample to return the same amount of 414Symbiodinium information, thus enabling more samples to be sequenced per unit cost.

415As part of optimising the SYM_VAR amplification protocol we tested whether increasing 416annealing temperature could increase the primers' taxonomic specificity for Symbiodinium. An 417increase in annealing temperature from $56^{\circ} \mathrm{C}$ to $59^{\circ} \mathrm{C}$ resulted in a higher proportion of 418Symbiodinium being amplified (Fig. 3). However, this was accompanied by a minor increase in 419the proportion of clade D Symbiodinium amplified. Given that any change in eladatclade 420proportion resulting from an increased annealing temperature is most likely due to preferential 421amplification of certain clades over others, this increase may be symptomatic of a weak 
422 ladatclade bias. Considered alongside the fact that sufficient Symbiodinium sequences were 423amplified to enable a robust analysis in the $\mathrm{CO}, \mathrm{CSW}$, and SRF-CO samples at $56^{\circ} \mathrm{C}$, and that 424increasing annealing temperature did not enable the amplification of Symbiodinium DNA in the 425SRF-OO $\Theta A$ samples, we recommend the $56^{\circ} \mathrm{C}$ annealing temperature to preference426minimisingminimise eladatclade bias at the expense of additional Symbiodinium amplification. 427Notably, although every effort has been made to design the SYM VAR primer pair in a manner 428that minimises Symbiodinium eladatclade bias, further testing for eladalclade bias in particular 429when working with Symbiodinium clades not recovered in this study may be needed to 430unequivocally confirm minimal bias. To this end, amplification, sequencing and analysis of pre431determined, artificially mixed communities of Symbiodinium may offer an effective way to test 432for eladatclade-bias.

433

434We also tested whether a reduced number of PCR cycles could still effectively amplify 435Symbiodinium DNA and whether the cycle number would affect chimeric sequence production. 436Although no Symbiodinium amplification was achieved in the 2 SRF-OOӨA samples tested, $\underline{\text { a }}$ 437greaterinereased amplification of Symbiodinium at 35 rather than 25 cycles in the CSW and SRF= 438 CO samples (Fig. 3), accompanied by only minimal reductioninerease in chimeric sequence 439production (Fig. 2) at the lower cycles,z), advocates the use of the higher cycles in the 440standardised protocol. Notably, any cycles required for the preparation of NGS libraries should 441be included in the number of total cycles. For example, if adapter and index attachment require a 442total of 7 cycles, then 28 cycles should be used for the initial PCR. Otherwise 35 cycles should be 443used for non-NGS applications. 
444Finally, we investigated whether the polymerase system used could modify the specificity of the 445SYM_VAR pair. Importantly, our results showed that the use of the Advantage 2 rather than the 446Phusion system led to a decreased specificity for Symbiodinium DNA when used in conjunction 447with the SYM_VAR pair (Fig. 4). Whilst it is not our objective to recommend the use of one 448polymerase system over any other, and it is likely unrealistic to expect research groups to diverge 449from polymerase systems established in their labs, our results do highlight that thea polymerase 450system used can have an effect on specificity and should therefore be taken into consideration 451during PCR optimisations. Reassuringly, the profile of ITS2 sequences returned from each of the 452polymerase systems were comparable (Fig 2). This would suggest that although polymerase 453system may affect the efficiency of amplification of Symbiodinium, it will have no effect on 454inferences made from data.

455Alongside considerations of Symbiodinium amplification efficiency (i.e., whether Symbiodinium 456can be amplified), it is also important to assess amplifications in terms of what taxonomic 457inferences they may offer. Due to its multicopy nature and the circumstance that more than one 458Symbiodinium taxa can be associated with any given host, the level of taxonomic resolution 459offered by the ITS2 genetic marker within Symbiodinium is dependent on the depth of 460sequencing. As the returned number of Symbiodinium sequences decreases, so does the power to 461resolve. When 100s to 1000s of sequences (per clade) are returned, ITS2 type profiles (sets of 462intragenomic sequences diagnostic of Symbiodinium taxa) can be assessed in detail (Smith et al. 4632017). Analyses that make use of intragenomic variance in such a way are very recent or still in 464development. Thus, species descriptions have yet to make use of NGS-derived ITS2 type 465profiles, but their identification has the ability to resolve at, and beyond, the species level within 
466Symbiodinium_(Hume et al. 2015; LaJeunesse et al. 2014; Smith et al. 2017).: Whilst all of the 467primer pairs were able to return such sequencing depth in the CO and CSW samples, for the SRF468 $\underline{\mathrm{CO}}$ and SRF-OOOA samples this depth was only achieved by the SYM_VAR primer pair (and 469not consistently across all samples). With this resolution, correlations between in hospite and 470free-living Symbiodinium populations can be addressed with a high degree of certainty. Notably, 471the level of sequencing depth becomes rather an issue of sequencing effort. Hence, differences in 472sequencing efforts for different environments will need to be taken into account if comparisons 473are planned to be made between different environments at the same resolution.

474

475Conclusions

476Given the ongoing popularity and increasing interest in phylotyping Symbiodinium in host as well 477as non-host environments employing the ITS2 region, we set out to develop a standardised 478Symbiodinium ITS2 amplification protocol, in particular for the use of NGS approaches. We 479show that the SYM_VAR_5.8S2/SYM_VAR_REV primer pair represent a superior primer pair 480able to amplify a greater proportion of Symbiodinium DNA with minimal cladal bias in 481comparison to other commonly used primers. In particular, the SYM_VAR primer pair's's ability 482to amplify from Symbiodinium-rare environments whilst maintaining maximum taxonomic 483resolving power holds potential for better elucidating the role of free-living Symbiodinium in reef 484ecology. Our standardised protocol should therefore help to-maximize return of information from 485a diverse range of sample types whilst maintaining comparability to future and existing projects. 


\section{Acknowledgements}

488This project has been funded through the Tara consortium, France Genomique grant number 489ANR-10-INBS-09, and KAUST baseline and KAUST BESE division funds to CRV.

490TaraTARA Pacific consortium acknowledgement: We are keen to thank the commitment of the 491people and the following institutions and sponsors who made this singular expedition possible: 492CNRS, CSM, PSL, KAUST, Genoscope/CEA, ANR-CORALGENE, France Genomique 493(specifically grant number ANR-10-INBS-09), agnès b., the Veolia Environment Foundation, 494Region Bretagne, Serge Ferrari, Billerudkorsnas, AmerisourceBergen Company, Lorient 495Agglomeration, Oceans by Disney, the Prince Albert II de Monaco Foundation, L’Oréal, 496Biotherm, France Collectivités, Kankyo Station, Fonds Français pour l’Environnement Mondial 497(FFEM), Etienne Bourgois, UNESCO-IOC, the Tara Foundation teams and crew. Tara Pacific 498would not exist without the continuous support of the participating institutes. This work is 499contribution number 4 of Tara Pacific. 


\section{References}

501Alberti A, Poulain J, Engelen S, Labadie K, Romac S, Ferrera I, Albini G, Aury JM, Belser C, 502 Bertrand A, Cruaud C, Da Silva C, Dossat C, Gavory F, Gas S, Guy J, Haquelle M, 503 Jacoby E, Jaillon O, Lemainque A, Pelletier E, Samson G, Wessner M, Acinas SG, Royo504 Llonch M, Cornejo-Castillo FM, Logares R, Fernandez-Gomez B, Bowler C, Cochrane 505 G, Amid C, Ten Hoopen P, De Vargas C, Grimsley N, Desgranges E, Kandels-Lewis S, 506 Ogata H, Poulton N, Sieracki ME, Stepanauskas R, Sullivan MB, Brum JR, Duhaime

507 MB, Poulos BT, Hurwitz BL, Pesant S, Karsenti E, Wincker P, Team GT, and 508 Consortium TO. 2017. Viral to metazoan marine plankton nucleotide sequences from the

509 Tara Oceans expedition. Scientific Data 4. ARTN 170093 10.1038/sdata.2017.93

510Arif C, Daniels C, Bayer T, Banguera-Hinestroza E, Barbrook A, Howe CJ, Lajeunesse TC, and $511 \quad$ Voolstra CR. 2014. Assessing Symbiodinium diversity in scleractinian corals via next512 generation sequencing-based genotyping of the ITS2 rDNA region. Molecular Ecology $513 \quad$ 23:4418-4433. 10.1111/mec.12869

514Boulotte NM, Dalton SJ, Carroll AG, Harrison PL, Putnam HM, Peplow LM, and van Oppen 515 MJH. 2016. Exploring the Symbiodinium rare biosphere provides evidence for symbiont 516 switching in reef-building corals. Isme Journal 10:2693-2701. 10.1038/ismej.2016.54

517Bourne DG, Morrow KM, and Webster NS. 2016. Insights into the Coral Microbiome:

$518 \quad$ Underpinning the Health and Resilience of Reef Ecosystems. Annual Review of

519 Microbiology, Vol 70 70:317-340. 10.1146/annurev-micro-102215-095440

520Coleman AW, Suarez A, and Goff LJ. 1994. Molecular Delineation of Species and Syngens in 521 Volvocacean Green-Algae (Chlorophyta). Journal of Phycology 30:80-90. DOI 522 10.1111/j.0022-3646.1994.00080.x

523Cunning R, Gates RD, and Edmunds PJ. 2017. Using high-throughput sequencing of ITS2 to 524 describe Symbiodinium metacommunities in St. John, US Virgin Islands. Peerj 5. ARTN $525 \quad$ e347210.7717/peerj.3472

526Hughes TP, Barnes ML, Bellwood DR, Cinner JE, Cumming GS, Jackson JBC, Kleypas J, van 527 de Leemput IA, Lough JM, Morrison TH, Palumbi SR, van Nes EH, and Scheffer M. 528 2017. Coral reefs in the Anthropocene. Nature 546:82-90. 10.1038/nature22901

529Hume B, D'Angelo C, Burt J, Baker AC, Riegl B, and Wiedenmann J. 2013. Corals from the $530 \quad$ Persian/Arabian Gulf as models for thermotolerant reef-builders: Prevalence of clade C3 531 Symbiodinium, host fluorescence and ex situ temperature tolerance. Marine Pollution $532 \quad$ Bulletin 72:313-322. 10.1016/j.marpolbul.2012.11.032

533Hume BCC. 2013. Are specific coral-Symbiodinium partnerships associated with survival in 534 extreme temperature environments of the Persian/Arabian Gulf? PhD thesis. University of 535 Southampton.

536Hume BCC, D'Angelo C, Smith EG, Stevens JR, Burt J, and Wiedenmann J. 2015.

537 Symbiodinium thermophilum sp nov., a thermotolerant symbiotic alga prevalent in corals 
538 of the world's hottest sea, the Persian/Arabian Gulf. Scientific Reports 5.

$539 \quad 10.1038 /$ srep08562

540Hume BCC, Voolstra CR, Arif C, D'Angelo C, Burt JA, Eyal G, Loya Y, and Wiedenmann J. 5412016 . Ancestral genetic diversity associated with the rapid spread of stress-tolerant coral

542 symbionts in response to Holocene climate change. Proceedings of the National Academy

543 of Sciences of the United States of America 113:4416-4421. 10.1073/pnas.1601910113

544Kemp DW, Hernandez-Pech X, Iglesias-Prieto R, Fitt WK, and Schmidt GW. 2014. Community

545 dynamics and physiology of Symbiodinium spp. before, during, and after a coral

$546 \quad$ bleaching event. Limnology and Oceanography 59:788-797. 10.4319/lo.2014.59.3.0788

547LaJeunesse TC. 2001. Investigating the biodiversity, ecology, and phylogeny of endosymbiotic

548 dinoflagellates in the genus Symbiodinium using the its region: In search of a "species"

549 level marker. Journal of Phycology 37:866-880. DOI 10.1046/j.1529-8817.2001.01031.x

550LaJeunesse TC. 2002. Diversity and community structure of symbiotic dinoflagellates from

551 Caribbean coral reefs. Marine Biology 141:387-400. 10.1007/s00227-002-0829-2

552LaJeunesse TC, Wham DC, Pettay DT, Parkinson JE, Keshavmurthy S, and Chen CA. 2014.

$553 \quad$ Ecologically differentiated stress-tolerant endosymbionts in the dinoflagellate genus

$554 \quad$ Symbiodinium (Dinophyceae) Clade D are different species. Phycologia 53:305-319.

$555 \quad$ 10.2216/13-186.1

556Levin RA, Voolstra CR, Weynberg KD, and van Oppen MJH. 2017. Evidence for a role of

$557 \quad$ viruses in the thermal sensitivity of coral photosymbionts. Isme Journal 11:808-812.

$558 \quad$ 10.1038/ismej.2016.154

559Moberg F, and Folke C. 1999. Ecological goods and services of coral reef ecosystems.

$560 \quad$ Ecological Economics 29:215-233. Doi 10.1016/S0921-8009(99)00009-9

561Mordret S, Romac S, Henry N, Colin S, Carmichael M, Berney C, Audic S, Richter DJ, Pochon

562 X, de Vargas C, and Decelle J. 2016. The symbiotic life of Symbiodinium in the open

563 ocean within a new species of calcifying ciliate (Tiarina sp.). Isme Journal 10:1424-1436.

$564 \quad$ 10.1038/ismej.2015.211

565Muscatine L. 1990. The role of symbiotic algae in carbon and energy flux in reef corals. In:

566 Dubinsky Z, ed. Ecosystems of the world 25: Coral Reefs. Amsterdam: Elsevier.

567Nitschke MR, Davy SK, and Ward S. 2016. Horizontal transmission of Symbiodinium cells

568 between adult and juvenile corals is aided by benthic sediment. Coral Reefs 35:335-344.

$569 \quad$ 10.1007/s00338-015-1349-0

570Norstrom AV, Nystrom M, Jouffray JB, Folke C, Graham NAJ, Moberg F, Olsson P, and

571 Williams GJ. 2016. Guiding coral reef futures in the Anthropocene. Frontiers in Ecology

$572 \quad$ and the Environment 14:490-498. 10.1002/fee.1427

573Pandolfi JM, Connolly SR, Marshall DJ, and Cohen AL. 2011. Projecting Coral Reef Futures

$574 \quad$ Under Global Warming and Ocean Acidification. Science 333:418-422.

$575 \quad$ 10.1126/science.1204794 
576Peixoto RS, Rosado PM, Leite DCD, Rosado AS, and Bourne DG. 2017. Beneficial

$577 \quad$ Microorganisms for Corals (BMC): Proposed Mechanisms for Coral Health and

$578 \quad$ Resilience. Frontiers in Microbiology 8. ARTN 34110.3389/fmicb.2017.00341

579Pesant S, Not F, Picheral M, Kandels-Lewis S, Le Bescot N, Gorsky G, Iudicone D, Karsenti E,

$580 \quad$ Speich S, Trouble R, Dimier C, Searson S, and Consortium TO. 2015. Open science

581 resources for the discovery and analysis of Tara Oceans data. Scientific Data 2. ARTN

$582 \quad 15002310.1038 /$ sdata.2015.23

583Plaisance L, Caley MJ, Brainard RE, and Knowlton N. 2011. The Diversity of Coral Reefs: What $584 \quad$ Are We Missing? Plos One 6. 10.1371/journal.pone.0025026

585Pochon X, and Gates RD. 2010. A new Symbiodinium clade (Dinophyceae) from soritid

586 foraminifera in Hawai'i. Molecular Phylogenetics and Evolution 56:492-497.

$587 \quad$ 10.1016/j.ympev.2010.03.040

588Pochon X, Pawlowski J, Zaninetti L, and Rowan R. 2001. High genetic diversity and relative

589 specificity among Symbiodinium-like endosymbiotic dinoflagellates in soritid

590 foraminiferans. Marine Biology 139:1069-1078.

591Pochon X, Putnam HM, and Gates RD. 2014. Multi-gene analysis of Symbiodinium

592 dinoflagellates: a perspective on rarity, symbiosis, and evolution. Peerj 2. ARTN

593 e39410.7717/peerj.394

594Pochon X, Stat M, Takabayashi M, Chasqui L, Chauka LJ, Logan DDK, and Gates RD. 2010.

$595 \quad$ Comparison of Endosymbiotic and Free-Living Symbiodinium (Dinophyceae) Diversity

596 in a Hawaiian Reef Environment. Journal of Phycology 46:53-65. 10.1111/j.1529-

597 8817.2009.00797.x

598Pogoreutz C, Radecker N, Cardenas A, Gardes A, Voolstra CR, and Wild C. 2017. Sugar

599 enrichment provides evidence for a role of nitrogen fixation in coral bleaching. Global

$600 \quad$ Change Biology 23:3838-3848. 10.1111/gcb.13695

601Radecker N, Pogoreutz C, Voolstra CR, Wiedenmann J, and Wild C. 2015. Nitrogen cycling in 602 corals: the key to understanding holobiont functioning? Trends in Microbiology 23:490-

603 497. 10.1016/j.tim.2015.03.008

604Rowan R, Knowlton N, Baker A, and Jara J. 1997. Landscape ecology of algal symbionts creates

605 variation in episodes of coral bleaching. Nature 388:265-269. Doi 10.1038/40843

606Schloss PD, Westcott SL, Ryabin T, Hall JR, Hartmann M, Hollister EB, Lesniewski RA, Oakley 607 BB, Parks DH, Robinson CJ, Sahl JW, Stres B, Thallinger GG, Van Horn DJ, and Weber

608 CF. 2009. Introducing mothur: Open-source, platform-independent, community-supported

609 software for describing and comparing microbial communities. Applied and

610 Environmental Microbiology 75:7537-7541. 10.1128/Aem.01541-09

611Silverstein RN, Cunning R, and Baker AC. 2015. Change in algal symbiont communities after 612 bleaching, not prior heat exposure, increases heat tolerance of reef corals. Global Change

613 Biology 21:236-249. 10.1111/gcb.12706 
614Smith EG, Ketchum RN, and Burt JA. 2017. Host specificity of Symbiodinium variants revealed 615 by an ITS2 metahaplotype approach. ISME J 11:1500-1503. 10.1038/ismej.2016.206

616Stat M, Pochon X, Cowie ROM, and Gates RD. 2009. Specificity in communities of

617 Symbiodinium in corals from Johnston Atoll. Marine Ecology Progress Series 386:83-96.

$618 \quad 10.3354 /$ meps08080

619Thomas L, Kendrick GA, Kennington WJ, Richards ZT, and Stat M. 2014. Exploring

620 Symbiodinium diversity and host specificity in Acropora corals from geographical

621 extremes of Western Australia with 454 amplicon pyrosequencing. Molecular Ecology

$622 \quad$ 23:3113-3126. 10.1111/mec.12801

623Thornhill DJ, Howells EJ, Wham DC, Steury TD, and Santos SR. 2017. Population genetics of 624 reef coral endosymbionts (Symbiodinium, Dinophyceae). Molecular Ecology 26:2640-

$625 \quad$ 2659. 10.1111/mec.14055

626Torda G, Donelson JM, Aranda M, Barshis DJ, Bay L, Berumen ML, Bourne DG, Cantin N, 627 Foret S, Matz M, Miller DJ, Moya A, Putnam HM, Ravasi T, van Oppen MJH, Thurber 628 RV, Vidal-Dupiol J, Voolstra CR, Watson SA, Whitelaw E, Willis BL, and Munday PL. 629 2017. Rapid adaptive responses to climate change in corals. Nature Climate Change $630 \quad$ 7:627-636. 10.1038/Nclimate3374

631Wham DC, Ning G, and LaJeunesse TC. 2017. Symbiodinium glynnii sp nov., a species of 632 stress-tolerant symbiotic dinoflagellates from pocilloporid and montiporid corals in the 633 Pacific Ocean. Phycologia 56:396-409. 10.2216/16-86.1

634Ziegler M, Arif C, Burt JA, Dobretsov S, Roder C, LaJeunesse TC, and Voolstra CR. 2017a. 635 Biogeography and molecular diversity of coral symbionts in the genus Symbiodinium 636 around the Arabian Peninsula. Journal of Biogeography 44:674-686. 10.1111/jbi.12913

637Ziegler M, Eguíluz VM, Duarte CM, and Voolstra CR. 2017b. Rare symbionts may contribute to 638 the resilience of coral-algal assemblages. The Isme Journal. 10.1038/ismej.2017.151

639Ziegler M, Seneca FO, Yum LK, Palumbi SR, and Voolstra CR. 2017c. Bacterial community 640 dynamics are linked to patterns of coral heat tolerance. Nature Communications 8. ARTN $641 \quad 1421310.1038 /$ ncomms14213

642 
643Gonfliet of interest

644The authors declare no conflict of interest.

645Data accessibility

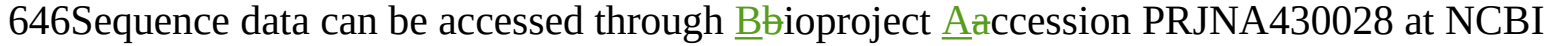

647(https://wwebi.nlm.nih.gov/https://www.ncbi.nlm.nih.gov/bioproject/PRJNA430028).

648Author Gontributions

649Goneeived and designed the experiments: BCEH, MZ, JP, PW, CRV; generated data, provided650tools/reagents/samples: BCCH, MZ, JP, XP, SR, EB, CdV, SP, PW, CRV; analysed data: BCCH, 651MZ, JP, CRV; wrote manuscript: BCCH, MZ, CRV; revised manuscript: BCCH, MZ, JP, XP, 652PW, CRV

653Sampling permits

654Panama scientific sampling: SE/AP-18-16; Panama CITES: SEX/A-72-16, no. 05567; 655Portulgal scientific sampling: 080522/2009/Proc. ${ }^{\circ}$ E.17.b; Maldives scientific sampling: FA656D2/33/2010/02; Chile scientific sampling: 13270/24/74 


\section{Table headers}

658

659Table 1 Primer pairs tested.

660

661Table 2 Sample overview and PCR conditions. Samples analysed for primer pair comparisons 662and optimisations are denoted.

663

664Table 3 Effect of number of PCR cycles on chimeric amplicon formation.

665 


\section{Figure legends}

667Figure 1 Comparison and taxonomic composition of sequences returned using three 668Symbiodinium ITS2 primer pairs. Each plot represents one sample and contains three pairs of 669stacked columns, one for each primer pair in the order of: SYM_VAR, ITSintfor, ITS-DINO 670 (from left to right). For each pair of stacked columns, the left coloured bar denotes the taxonomic 671breakdown of all returned sequences into 7 categories, and the right greyscale bar denotes the 672sub-genus (cladal) distribution for the subset of sequences classified as Symbiodinium (see colour 673legend). Plots are annotated with the number of sequences returned after QC and the proportion 674of those sequences that were annotated as Symbiodinium underneath the coloured and grayscale 675bars, respectively. For each environment type (indicated to the left of the plot groupings) a 676summary plot is given at the right side, showing the average taxonomic breakdowns, total post677QC sequences returned, and average proportion of Symbiodinium sequences.

678Figure 2 Robustness of PCR protocol using the SYM_VAR ITS2 primer pair. Each subplot 679represents a pairwise comparison of PCRs from the same sample. The sample, environment type, 680and PCR conditions are noted above each subplot. Depending on which pair of PCRs are being 681compared, these subplots graphically represent effects due to ( $\underline{\mathrm{A}} \mathrm{t})$ PCR-to-PCR variation, $(\underline{B} z)$ 682annealing temperature, (C 3 ) number of PCR cycles, or (D4) polymerase system used. For each 683subplot, the relative presence of the 20 most abundant amplicons (calculated across both PCRs) is 684plotted for each PCR, denoted as black and grey bars. For example, the top left subplot of the 685figureAii represents a pairwise comparison of PCRs run using the same annealing temp and 686number of cycles on sample AW-0000035. It is therefore testing the PCR-to-PCR effect.

687Figure 3 Effect of annealing temperature and number of PCR cycles on sequences returned 688using the SYM_VAR ITS2 primer pair. Two annealing temperatures (a; 56 and $59^{\circ} \mathrm{C}$; 689columns) and two cycle numbers (b; 25 and 35 cycles; columns) were tested on samples from 690three different environment types (rows; indicated to the left of the plot groupings). Each plot 691represents a different pooled PCR amplicon with sample name, sample type, number of cycles, 692and annealing temperature noted to the left. For each plot, coloured bars on the left denote the 693taxonomic breakdown of all returned sequences into 7 categories. The right greyscale bars denote 694the sub-genus (cladal) distribution for the subset of sequences classified as Symbiodinium. Plots 695are annotated with the number of sequences returned after QC and the proportion of those 696sequences that were annotated as Symbiodinium underneath the coloured and grayscale bars, 697respectively. Symbiodinium-derived sequences were further subcategorized according to their 698cladal identity (greyscale stacked bars).

\section{Figure 4 Effect of polymerase type on sequences returned using the SYM_VAR ITS2}

700primer pair. Each plot represents one sample and contains two pairs of stacked columns, one for 701each polymerase with Phusion (ThermoFisher Scientific, USA) on the left, and Advantage 2 702(Takara bio, Japan) on the right. Coloured bars denote the taxonomic breakdown of all returned 703 sequences into 7 categories. The greyscale bars denote the sub-genus (cladal) distribution for the 704subset of sequences classified as Symbiodinium. Plots are annotated with the number of 705sequences returned after QC and the proportion of those sequences that were annotated as 706Symbiodinium underneath the coloured and grayscale bars, respectively. Plots are ordered by 
707environment (rows; CO - coral, CSW - coral surrounding water, SRF-CO - surface water, SRF708OOOA - open ocean). A summary plot is provided for each environment type at the end of the 709row.

710 


\section{Supplemental Information}

712Supplementary Table 1 Sample details and PCR conditions. Samples analysed for primer pair 713comparisons and optimisations are denoted.

\section{4}

715Supplementary Figure 1. Mean sequences returned per sample and proportion retained 716 during the quality control pipeline (QC) in relation to primer pair used. The number of 717sequences retained at three stages of the QC pipeline are given: 'raw contigs' (number of 718sequences returned from mothur's make.contigs command), 'post-qc' (the number of sequences 719remaining after all quality control and taxonomic identification), and 'Symbiodinium' (the 720 number of sequences identified as Symbiodinium). Samples are grouped according to their 721environment and amplification primers. Abbreviations as follows: CO - coral tissue, CSW 722coral surrounding water, SRF-CO - coral reef surface water, SRF-OO - open ocean surface 723 water.

\section{4}

725Supplementary Figure 2. Comparison and taxonomic composition of sequences returned 726using three Symbiodinium ITS2 primer pairs before removal of sequences JN406302 and 727JN406301. Each plot represents one sample and contains three pairs of stacked columns, one for 728each primer pair in the order of: SYM_VAR, ITSintfor, ITS-DINO (from left to right). For each 729pair of stacked columns, the left coloured bar denotes the taxonomic breakdown of all returned 730sequences into 7 categories, and the right greyscale bar denote the sub-genus (cladal) distribution 731for the subset of sequences classified as Symbiodinium. Plots are annotated with the number of 732sequences returned after QC and the proportion of those sequences that were annotated as 733Symbiodinium underneath the coloured and grayscale bars, respectively. For each environment 734type (indicated to the left of the plot groupings) a summary plot is given at the right side, 735showing the average taxonomic breakdowns, total post-QC sequences returned, and average 736proportion of Symbiodinium sequences.

\section{7}

738

739Supplementary Data 1 - The fasta sequences used to generate the Symbiodinium cladal 740 database. 